\title{
A Framework for Computing Bounds for the Return of a Policy
}

\author{
Cosmin Păduraru, Doina Precup, and Joelle Pineau \\ McGill University, School of Computer Science, Montreal, Canada
}

\begin{abstract}
We present a framework for computing bounds for the return of a policy in finite-horizon, continuous-state Markov Decision Processes with bounded state transitions. The state transition bounds can be based on either prior knowledge alone, or on a combination of prior knowledge and data. Our framework uses a piecewise-constant representation of the return bounds and a backwards iteration process. We instantiate this framework for a previously investigated type of prior knowledge - namely, Lipschitz continuity of the transition function. In this context, we show that the existing bounds of Fonteneau et al. $(2009,2010)$ can be expressed as a particular instantiation of our framework, by bounding the immediate rewards using Lipschitz continuity and choosing a particular form for the regions in the piecewise-constant representation. We also show how we can improve upon their bounds by modifying these choices.
\end{abstract}

\section{Introduction}

We are interested in reinforcement learning (RL) problems that arise in domains where guaranteeing a certain minimum level of performance is important. In domains such as medicine, engineering, or finance, there are often large material or ethical costs associated with executing a policy that performs poorly. In order for RL to have a positive impact on these areas, it is therefore necessary to find ways to guarantee that the policy produced by an RL algorithm will achieve a certain level of performance.

The performance of a policy is measured via its return, the (possibly discounted) sum of rewards obtained when executing the policy. The return is a random variable, and its value can change from one execution to the next depending on the stochasticity in the environment. In order to establish guarantees for the performance of a policy, many researchers (e.g., Brunskill et al. (2008) or Kakade et al., (2003) ) have developed bounds on the value function, which is the expected value of the return.

While it is undoubtedly useful to have information about a policy's value function, there may well be problems where we want to know more than the average performance - for instance, problems where the policy will only be executed a small number of times. In such problems, it can be useful to have a worst-case analysis for the return that the policy may obtain during its execution. This paper presents a framework for performing such an analysis.

Our framework transforms guarantees about the effect of individual transitions into guarantees about the return. For this, we require a human expert to either provide information about the range of each transition's outcomes, or to provide some regularity 
conditions that can then be used together with transition data to infer these ranges. For infinite state spaces, this prior knowledge would obviously have to be expressed in a compact form. We also require information about the range of immediate rewards that can be obtained from states in a certain region. Our framework transforms the information about the transition and reward function into bounds on the policy's return.

The framework can handle either discrete or continuous state spaces. In order to handle continuous state spaces, we use a piecewise-constant representation for the bounds. Other representations could have been chosen, but the piecewise-constant representation has the advantage that the prior knowledge about the transitions can to be expressed in a relatively simple manner - an important feature when working with human experts.

We show how the framework can be instantiated for a particular type of prior knowledge (Lipschitz continuity of the transition model) that has already been investigated by Fonteneau et al. (2009, 2010). The fact that transition and reward bounds can simply be plugged into our framework, rather than relying on a less flexible closed-form solution, allows us to identify algorithmic choices that can perform better in practice than the bounds of Fonteneau et al.

\section{Framework description}

We are concerned with decision-making problems where $S \subset \mathbb{R}^{d}$ is the set of states, $A \subset \mathbb{R}$ is the set of actions, $f: S \times A \rightarrow S$ is the transition function and $r: S \times A \rightarrow \mathbb{R}$ is the (deterministic) reward function. The transition function can be probabilistic, in which case $f(s, a)$ is a random variable. Our goal is to compute lower bounds ${ }^{1}$ for the return obtained by starting in some state $x_{0}$ and following the deterministic ${ }^{2}$ policy $\pi: S \rightarrow A$ for $K$ steps, where the return is defined as

$$
R_{K}^{\pi}\left(x_{0}\right)=\sum_{k=0}^{K-1} r\left(x_{k}, \pi\left(x_{k}\right)\right)
$$

s.t. $\forall k>0, x_{k} \sim f\left(x_{k-1}, \pi\left(x_{k-1}\right)\right)$.

Our central assumption is that we have a method for computing, given some region of the state space $\omega \subset S$, a region $f^{\pi}(\omega)$ such that

$$
\forall s \in \omega, f(s, \pi(s)) \in f^{\pi}(\omega) .
$$

Any choice of $f^{\pi}(\omega)$ that respects the above condition can be used, but the smaller the region produced, the tighter the final bounds will be. We will discuss possible methods for computing $f^{\pi}(\omega)$ further in the paper.

\footnotetext{
${ }^{1}$ We could easily extend our framework to compute upper bounds as well; while this may also have interesting applications, such as informing exploration strategies, we focus on lower bounds for now.

${ }^{2}$ Although we assume deterministic rewards and policies, these are not major restrictions. We can always add a state variable for probabilistic rewards, and make the reward function a deterministic function of that variable. In addition, the policies produced by many reinforcement learning methods are deterministic, and policies of interest in the target domains we envision are also usually deterministic.
} 
We also assume that we have a way to compute a lower bound for $\inf _{x \in \omega} r(x, \pi(x))$ for any region $\omega \subset S$. The quality of the final bounds will depend on the tightness of this lower bound for the immediate reward. Since $r$ and $\pi$ are typically known, we should be able to get informative lower bounds for most problems.

Let $\left\{\Omega_{k} \mid k=0, \ldots K\right\}$ be sets of partitions indexed by $k$. The lower bound for each $k$-step return $R_{k}^{\pi}$ will be represented as a function of the partitions in $\Omega_{K-k}$ (the indexing of $R_{k}^{\pi}$ increases with the horizon, whereas the indexing of $\Omega_{k}$ increases with the time step). For $s \in S$, we will write $s \in \Omega_{k}$ as a shorthand for $\exists \omega \in \Omega_{k}$ s.t. $s \in \omega$.

We are now ready to describe our algorithmic framework. We will use the following recursive algorithm for computing a lower bound for $R_{K}^{\pi}$ :

\section{Algorithm 1 Recursive lower bound for the K-step return}

1. For each $\omega \in \Omega_{K}$, let $\hat{R}_{0}^{\pi}(\omega)=\inf _{x \in \omega} r(x, \pi(x))$

2. Loop through $k=1, \ldots K$

For each $\omega \in \Omega_{K-k}$, compute the lower bound $\hat{R}_{k}^{\pi}(\omega)$ using

$$
\hat{R}_{k}^{\pi}(\omega)=\inf _{x \in \omega} r(x, \pi(x))+\inf _{\left\{\omega^{\prime} \in \Omega_{K-k+1} \mid \omega^{\prime} \cap f^{\pi}(\omega) \neq 0\right\}} \hat{R}_{k-1}^{\pi}\left(\omega^{\prime}\right)
$$

The following result shows that Algorithm 1 produces valid lower bounds for the $K$-step return.

Theorem 1. For any $k=0, \ldots K$, any $\omega \in \Omega_{K-k}$, and any $s \in \omega$ we have that

$$
\hat{R}_{k}^{\pi}(\omega) \leq R_{k}^{\pi}(s)
$$

Proof. By induction over $k$.

For $k=0$, the proof is immediate given the construction of $\hat{R}_{0}^{\pi}$.

For $k \rightarrow k+1$, let $\omega \in \Omega_{K-k}$ and $s \in \omega$. We have that

$$
R_{k+1}^{\pi}(s) \geq r(s, \pi(s))+\inf _{s^{\prime} \sim f(s, \pi(s))} R_{k}^{\pi}\left(s^{\prime}\right)
$$

Because $s \in \omega$ we have

$$
r(s, \pi(s)) \geq \inf _{x \in \omega} r(x, \pi(x))
$$

and

$$
f(s, \pi(s)) \subset f^{\pi}(\omega),
$$

which, together with the induction hypothesis and the update equation in Step 3 of the algorithm, lead to the conclusion that $\hat{R}_{k+1}^{\pi}(\omega) \leq R_{k+1}^{\pi}(s)$.

One can obtain different instantiations of the general framework described in Algorithm 1 depending on what choices are made for computing $f^{\pi}(\omega)$, for representing the partition sets $\Omega_{k}$, and for computing $\inf _{x \in \omega} r(x, \pi(x))$. In Section 3 we will use an idea from the work of Fonteneau et al. $(2009,2010)$ in order to compute $f^{\pi}(\omega)$ in the setting where the transition function is Lipschitz continuous. In the same section we will also propose two natural choices for representing the $\Omega_{k}$ sets and for computing $\inf _{x \in \omega} r(x, \pi(x))$. We will illustrate the empirical effect of those choices on two domains in Section 4. 


\section{Implementation for Lipschitz continuity}

In this section, we borrow ideas from Fonteneau et al. $(2009,2010)$ for computing $f^{\pi}(\omega)$ by incorporating prior knowledge in the form of Lipschitz continuity of the transition model.

\subsection{Notation and assumptions}

Let $S, A$, and $S \times A$ be normed spaces, and denote the distance metric on all of them by $d$ (it should be obvious from the context which one is being used). Define the distance between state-action pairs to be (Fonteneau et al.,2009):

$$
d\left(\left(s^{1}, a^{1}\right),\left(s^{2}, a^{2}\right)\right)=d\left(s^{1}, s^{2}\right)+d\left(a^{1}, a^{2}\right) .
$$

The transition function, reward and policy are said to be Lipschitz continuous if there exist constants $L_{f}, L_{r}$ and $L_{\pi}$ such that

$$
\begin{gathered}
d\left(f\left(s^{1}, a^{1}\right), f\left(s^{2}, a^{2}\right)\right) \leq L_{f} d\left(\left(s^{1}, a^{1}\right),\left(s^{2}, a^{2}\right)\right) \\
d\left(r\left(s^{1}, a^{1}\right), r\left(s^{2}, a^{2}\right)\right) \leq L_{r} d\left(\left(s^{1}, a^{1}\right),\left(s^{2}, a^{2}\right)\right)
\end{gathered}
$$

and

$$
d\left(\pi\left(s^{1}\right), \pi\left(s^{2}\right)\right) \leq L_{\pi} d\left(s^{1}, s^{2}\right)
$$

respectively, for all $s^{1}, s^{2} \in S$ and $a^{1}, a^{2} \in A$. The transition function is assumed to be deterministic.

In addition to Lipschitz continuity, we assume that there exists a set of $n$ observed transitions of the form $\left(s_{i}, a_{i}, r\left(s_{i}, a_{i}\right), f\left(s_{i}, a_{i}\right)\right)$, collected using an arbitrary and potentially unknown policy. Note that the actions in the batch do not have to match the actions taken by $\pi$, so this is an off-policy evaluation problem. Having this set of observed transitions allows them to bound the outcome $f(s, a)$ for any state-action pair $(s, a)$, by taking for some $i \in\{1, \ldots n\}$ a ball of center $f\left(s_{i}, a_{i}\right)$ and radius $L_{f} d\left((s, a),\left(s_{i}, a_{i}\right)\right)$, as illustrated in Figure 1 . We denote by $c_{\omega}$ the center and by $\rho_{\omega}$ the radius of a hypersphere containing $\omega$.

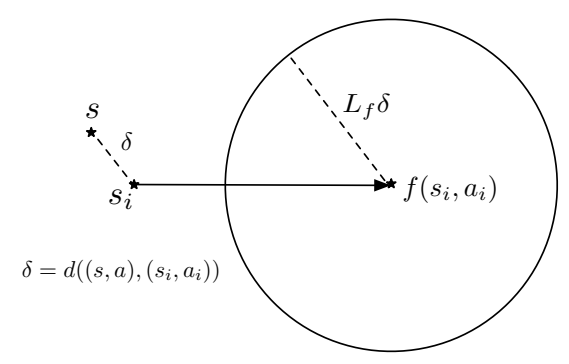

Fig. 1. Illustration of using Lipschitz continuity to bound $f(s, a)$. 


\subsection{Previous Work}

In their first paper, Fonteneau et al. (2009) provide a lower bound for finite-horizon value functions in Lipschitz-continuous deterministic systems with continuous state and action spaces. Given $K$ observed transitions (one for each step of the horizon), their bound has the form

$$
Q_{K}^{\pi}\left(x_{0}, \pi\left(x_{0}\right)\right) \geq \sum_{k=1}^{K}\left[r\left(s_{k}, a_{k}\right)-L_{Q}^{K-k}\left(d\left(s_{k}, f\left(s_{k-1}, a_{k-1}\right)\right)+d\left(a_{k}, \pi\left(f\left(s_{k-1}, a_{k-1}\right)\right)\right)\right],\right.
$$

where $L_{Q}$ is defined as

$$
L_{Q}^{K-k}=L_{r}\left(\sum_{i=0}^{K-k-1}\left[L_{f}\left(1+L_{\pi}\right)\right]^{i}\right)
$$

and is shown to be a valid Lipschitz constant for the value function.

In a second paper, Fonteneau et al. (2010) compute a similar bound for open-loop policies over discrete actions. This new bound can be derived from the one above if we replace $L_{\pi}$ and the distances between actions with zero. They use this bound to show how it can inform an algorithm for computing "cautious" policies that avoid unexplored regions of the state space. Since the main focus of this work is analyzing the tightness of bounds, we will not be concerned with their cautious generalization algorithm in this paper.

\subsection{Framework instantiation}

If the transition model is Lipschitz continuous and a batch of transitions is available, then one way of computing $f^{\pi}(\omega)$ is as follows:

1. Associate a state-action pair from the batch (denoted by $\left.\left(s_{\omega}, a_{\omega}\right)\right)$ to $\omega$. One heuristic way of doing this is by taking the closest state-action pair to $\left(c_{\omega}, \pi\left(c_{\omega}\right)\right)$. Another way, proposed by Fonteneau et al. (2009), is to search for the sequence of states that minimizes the $K$-step bound. In our experiments, we found that both methods performed similarly, but the method of Fonteneau et al. may well offer advantages in other settings.

2. Compute $f^{\pi}(\omega)$ as a ball of center $f\left(s_{\omega}, a_{\omega}\right)$ and radius

$$
L_{f}\left[d\left(c_{\omega}, s_{\omega}\right)+d\left(\pi\left(c_{\omega}\right), a_{\omega}\right)+\rho_{\omega}+\sup _{x \in \omega} d\left(\pi(x), \pi\left(c_{\omega}\right)\right)\right] .
$$

Using the definition of Lipschitz continuity and the triangle inequality we can easily show that, for $f^{\pi}(\omega)$ constructed in this fashion, the condition in Equation 2 holds.

In order to have a completely instantiated algorithm, we have to decide how to construct the $\Omega_{k}$, and how to compute $\inf _{x \in \omega} r(x, \pi(x))$ and $\sup _{x \in \omega} d\left(\pi(x), \pi\left(c_{\omega}\right)\right)$. Plugging these choices into Algorithm 1 we can obtain valid lower bounds for the return. 
Replicating the bounds of Fonteneau et al. Although Fonteneau et al. arrived at their results via a different approach, given a fixed set of $K$ transitions $\left(s_{i}, a_{i}, r\left(s_{i}, a_{i}\right), f\left(s_{i}, a_{i}\right)\right)$, $i=1, \ldots K$, their bound can be exactly replicated within our framework if we use the following choices in Algorithm 1:

- Have a single ball in each $\Omega_{k}$ As discussed earlier, and illustrated in Figure 1, bounding the outcomes of state transitions using Lipschitz continuity produces spherical bounds. Therefore, it is natural to consider each $\Omega_{k}$ to be composed of a single ball, starting with $\Omega_{0}=\left\{\omega_{0}\right\}$, where $\omega_{0}=\left\{x_{0}\right\}$, and then computing $\Omega_{k+1}=\left\{\omega_{k+1}\right\}=\left\{f^{\pi}(\omega)\right\}$ according to step 2 above.

- Bound policy changes using Lipschitz continuity They assume that $\pi$ is Lipschitz continuous, which allows the following upper bound to be constructed:

$$
\left.\sup _{x \in \omega} d\left(\pi(x), \pi\left(c_{\omega}\right)\right) \leq \sup _{x \in \omega} L_{\pi} d\left(x, c_{\omega}\right)\right)=L_{\pi} \rho_{\omega}
$$

- Bound rewards using Lipschitz continuity They assume that the reward is Lipschitz continuous, which allows for the bound

$$
\begin{aligned}
\inf _{x \in \omega} R(x, \pi(x)) & \geq r\left(s_{\omega}, a_{\omega}\right)-L_{r}\left[\sup _{x \in \omega}\left[d\left(s_{\omega}, x\right)+d\left(a_{\omega}, \pi(x)\right)\right]\right] \\
& \geq r\left(s_{\omega}, a_{\omega}\right)-L_{r}\left[d\left(s_{\omega}, c_{\omega}\right)+d\left(a_{\omega}, \pi\left(c_{\omega}\right)\right)+\rho_{\omega}+\sup _{x \in \omega} d\left(\pi(x), \pi\left(c_{\omega}\right)\right)\right]
\end{aligned}
$$

To see that the bounds are the same, note that, with the above choices and denoting $d(k)=d\left(s_{k}, f\left(s_{k-1}, a_{k-1}\right)\right)+d\left(a_{k}, \pi\left(f\left(s_{k-1}, a_{k-1}\right)\right)\right.$, each reward term in the lower bound of Algorithm 1 is lower bounded by

$$
r\left(s_{k}, a_{k}\right)-L_{r}\left[d(k)+\left(1+L_{\pi}\right) L_{f}\left(d(k-1)+\left(1+L_{\pi}\right) \rho_{k-1}\right)\right],
$$

where $\rho_{k-1}$ is the radius of the single ball in $\Omega_{k-1}$. When summing up all the terms, and after some re-arrangement, we obtain the bound of Fonteneau et al. (2009).

Note that the Fonteneau et al. (2010) paper considers blind policies (policies where the action at each stage does not depend on the state), so the distance between actions simply disappears in that bound.

Proposed alternatives We now consider two alternative algorithm instantiations that can lead to tighter bounds than previous results.

The first instantiation, which we will call single-ball-rmin, is similar to the Fonteneau et al. approach, except that it performs direct reward minimization instead of using Lipschitz continuity to bound the rewards. That is, we assume that we know $r$, and we use constraint optimization to optimize the reward within each ball. While this is not a big difference conceptually, it can make a large difference in practice. Also note that using our framework allows us to easily replace reward bounding using Lipschitz continuity with direct minimization. On the other hand, in the work of Fonteneau et al. the Lipschitz constant $L_{r}$ appears as a multiplicative term in their closed-form bounds, and there is no straightforward way to replace it with a local minimum of the reward function. 
The second instantiation will be called multi-partition-rmin, because it partitions each $\Omega_{k}$ into multiple regions. Using a single region for each $k$, as in the single-ball algorithm, has the disadvantage that it does not account for how $\pi$ may take different actions in different parts of $\Omega_{k}$. Therefore, in this algorithm we partition each $\Omega_{k}$; for computational simplicity, we will take the partition boundaries to be axis-parallel. The multi-partition algorithm also performs direct minimization for bounding the rewards.

For this paper, we will only consider the alternatives described above. However, we note that other interesting instantiations are also possible, an obvious one being to perform direct minimization of $\sup _{x \in \omega} d\left(\pi(x), \pi\left(c_{\omega}\right)\right)$ instead of using Lipschitz continuity. We leave such extensions for future work.

\subsection{Discussion of bounds based on Lipschitz continuity}

The tightness of the bounds produced by all the methods described above will depend on the value of the Lipschitz constants $L_{f}, L_{r}$ and $L_{\pi}$, which is related to the prior knowledge we have about the system and the characteristics of the domain. These values will influence all methods in a similar way. Another factor that influences all methods will be the distances $d\left(c_{\omega}, s_{\omega}\right)+d\left(\pi\left(c_{\omega}\right), a_{\omega}\right)$. As these distances get smaller, the bounds get tighter. The component $d\left(\pi\left(c_{\omega}\right), a_{\omega}\right)$, in particular, will depend on the ability to find transitions in the batch that take actions similar to those that $\pi$ would take at the centers of the $\omega$ regions. So, to sum up, the more transitions there are in the batch, and the closer the actions of those transitions are to the policy we are trying to evaluate, the tighter the bounds will be.

For the multi-partition method to be effective, we need the union of the $f^{\pi}(\omega)$ for $\omega \in \Omega_{k}$ to be small relative to the size of $f^{\pi}(\omega)$ that would be computed by the singleball algorithm. The size of the union relative to a single projection will depend on $\pi$, the policy we are evaluating. This influence of the nature of $\pi$ is difficult to express quantitatively, because $\pi$ can affect different parts of the state space in different ways. This difficulty in including more information about $\pi$ in a closed-form bound makes it difficult to predict what type of bound will perform better, but is also one of the main reasons for proposing the multi-partition method, since it is the only method that attempts to include such information.

Finally, the multi-partition bound will become tighter as the density of partitions in $\Omega$ increases. Because of this, the multi-partition method suffers from the curse of dimensionality when applied in its most basic form: the number of regions required to effectively cover the state space is exponential in the dimensionality of $S$, and the computational complexity of computing $f^{\pi}(\omega)$ for $\omega \in \Omega_{k}$, the most expensive part of the multi-partition algorithm, is $O\left(n *\left|\Omega_{k}\right| *\left|\Omega_{k+1}\right|\right)$. However, it should be the case in most problems that the policy $\pi$ that we are evaluating only visits a small region of the state space. In such problems, the forward projections computed using the multipartition method, and therefore the $\Omega_{k}$, are small relative to the size of the state space. In either case, the multi-partition method will be more computationally expensive than the single-ball method. We plan to explore these issues more thoroughly in future work. 


\section{Empirical results}

We now illustrate the proposed bounds and algorithmic choices in two sets of experiments.

\subsection{Puddle world}

For our first set of experiments, we consider the deterministic puddle world domain, as implemented by Fonteneau et al. (2010). The puddle world is a two-dimensional problem in which an autonomous agent has four discrete actions available, taking it in each of the cardinal directions. There are two regions (the puddles) where the agent receives negative rewards, and a goal region where the agent receives positive rewards. We use the same starting state as Fonteneau et al., and the same mechanism for generating an initial batch of transitions (we place them uniformly throughout the state space). Complete details for this implementation of puddle world can be found in Fonteneau et al. (2010). The policy $\pi$ that we evaluate is an open-loop policy that takes the agent around the puddle and to the goal region by going down for five steps, right for six steps, up for eight steps and right once again for the final step.

Fonteneau et al. (2010) use the bounds they compute as an intermediate part in their algorithm for computing cautiously generalizing policies. However, in this paper we are primarily concerned with the tightness of different types of bounds, so we will not carry out their experiment in full. Instead, we compare the bounds that are computed for $\pi$ by the method of Fonteneau et al. (2010) to the single-ball-rmin and multi-partition-rmin algorithms.

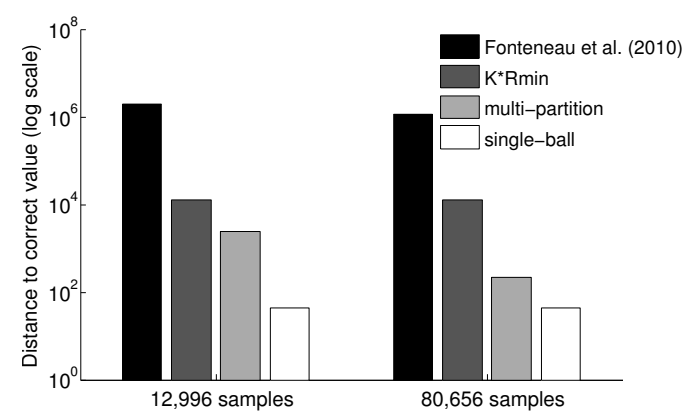

Fig. 2. Comparison of the tightness of different bounds on the puddle world domain. Smaller bars indicate tighter bounds.

The results, presented in Figure 2, show the tightness of the different bounds for two different sets of stored transitions. The starting states of the transitions were placed uniformly throughout the state space, as per Fonteneau et al. (2010), at distances of 0.05 along each axis for the first set of results and 0.02 along each axis for the second set of results. The multi-partition results shown in this figure are for 200,000 partitions in 
each $\Omega_{k}$. We also include the trivial bound obtained by multiplying the horizon by the smallest immediate reward (denoted by $K * R_{m}$ in on the graph).

The first thing to note is that, in this domain, the single-ball-rmin algorithm produces bounds that are the same order of magnitude as the actual return - its values for the two samples sizes are -98.09 and -81.74 , respectively, compared to a correct value of -54.08 . This happens because the domain is quite well-behaved, with $L_{f}=1$ and $L_{\pi}=0$ (meaning that the effect of the policy can be ignored, since the policy is openloop). The errors that accumulate over repeated projections are therefore solely due to the distances between the observed transitions and the centers of the projected regions.

The multi-partition-rmin algorithm is not performing as well as single-ball-rmin in this domain. Because the policy does not depend on the state, there is no advantage to incorporating information about how the policy changes in different parts of $\Omega_{k}$. Instead, the multi-partition algorithm produces looser bounds because of the extra approximations it needs to use in order to handle state-dependent policies.

Finally, because of the large value of $L_{r}$ in this domain $\left(1.3742 * 10^{6}\right)$, methods that lower bound the reward using Lipschitz continuity perform poorly. For instance, the bound computed by the Fonteneau et al. method using a sample size of 80656 transitions is equal to $-55.94 * 10^{4}$. In comparison, the trivial bound obtained by multiplying the smallest reward by the horizon is actually significantly tighter, having the value $-1.3 *$ $10^{4}$.

The main conclusions that we draw from this experiment is that the additional step of analytically minimizing the (known) immediate reward can offer significant improvements with respect to Lipschitz-continuity-based bounds, and that the multi-partition method is unlikely to be useful if the policy does not depend on the state.

\subsection{D problem with stabilizing policy}

In this section, we use a simple one-dimensional problem to illustrate that the multipartition approach can lead to tighter bounds when the policy depends on the state.

The domain we are using has $S=\mathbb{R}, A=\mathbb{R}, f(s, a)=s+a$ and $r(s, a)=-|s|$. We compute lower bounds for a policy that always takes the agent halfway to state 0 , that is, $\pi(s)=-s / 2$. We assume that we have a batch of observed transitions that was collected using a different policy, one for which the action at state $s$ is uniformly distributed in $[-s, 0]$. The starting states for the observed transitions are in the $[-1,1]$ interval, and the starting state for evaluating $\pi$ was -1 . The Lipschitz constants for the reward and transition function are equal to one, while $L_{\pi}=0.5$, so $L_{f}\left(1+L_{\pi}\right)=1.5$.

We compare the lower bound computed using the single-ball-rmin and multi-partitionrmin algorithms to the analytic bound of Fonteneau et al. (2009). The number of partitions in $\Omega$ for the multi-partition method was set to 1000 . The results are presented in Figure 3. The size of the batch was 1000 transitions, with the start state of those transitions generated uniformly randomly between -1 and 1 . The results were averaged over 50 trials, with all trials exhibiting the same pattern.

Note the logarithmic scale on the figure. The Fonteneau et al. (2009) single-ball bounds scale exponentially in the horizon $K$. This is due to the fact that a factor of $L_{f}\left(1+L_{\pi}\right)^{K}$ appears in their Lipschitz constant for the value function $L_{Q}$, which then gets multiplied by the distance between the end point of a transition and the start of 


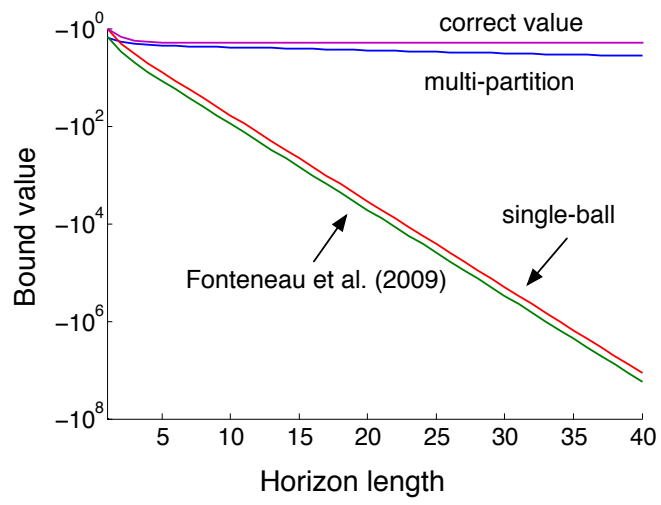

Fig. 3. Comparison of bound values for the one-dimensional problem for different horizon lengths.

another. A small improvement in performance can be observed when using the singleball-rmin algorithm instead of the method of Fonteneau et al. (2009). However, in this problem the effect of multiplying by an exponential function of $L_{f}\left(1+L_{\pi}\right)=1.5$ is still dominant with respect to the effect of bounding the reward using Lipschitz continuity.

The main point of this experiment was to illustrate that not accounting for statedependent policy effects can produce bounds that scale up exponentially in the horizon even for very simple problems, and that the multi-partition algorithm has the potential to alleviate this issue. While we acknowledge that the experiment was crafted specifically to highlight this issue, we believe that it is illustrative of settings that may arise in practical applications; for instance, in dynamical system control the controller is often designed so that it adjusts the system towards a particular desired state or range of states.

\section{Discussion and future work}

We proposed a framework for computing bounds on the return of a policy when knowledge about the environment is available in the form of bounds on the effect of the transition function. By doing this, we showed how to break the bound computation process down into simpler individual components.

We also instantiated our bound for the setting where the transition function, reward, and policy are Lipschitz continuous. In this setting, we showed how the results of Fonteneau et al. $(2009,2010)$ can be regarded as a particular instantiation of our framework, but we also illustrated ways in which making different choices for the framework's components allows us to produce potentially more informative bounds.

One limitation with respect to previous work is that, while Fonteneau et al. (2009) provide an algorithm for finding the best sequence of transitions to use, we do not perform such an optimization for our methods. Instead, we simply pick the $k$ th transition such that its starting state-action pair is the closest to $\left(f\left(s_{k-1}, a_{k-1}\right), \pi\left(f\left(s_{k-1}, a_{k-1}\right)\right)\right)$. For one of the methods we propose, the single-ball method, we noticed no difference 
between using this heuristic and performing the optimization in our experiments. For the multi-partition method, the optimization problem is more difficult and we do not address it here.

A key part of our framework is the computation of $f^{\pi}(\omega)$. In order to guarantee that $f^{\pi}(\omega)$ respects the property in Equation 2, we need to rely on some form of prior knowledge. In this paper, the type of prior knowledge we used was Lipschitz continuity of the transition function.

In general, the type of prior knowledge available can have increasing levels of sophistication. Let us take robot navigation as an example. A very simple form of prior knowledge would be that each action moves the robot by a fixed amount in some direction, plus some bounded noise. To this, one could add different types of terrain, with different levels of noise associated with each type; such an approach is taken by Brunskill et al. (2008), who produce bounds on the value function under the assumption that the transition function is Gaussian.

Further, the amount of noise could be learned from the data, based on regularity conditions about the noise not varying too much within certain regions. When using data to inform the transition bounds in stochastic systems, one typically obtains stochastic guarantees of the form "with probability $\alpha, s \in \omega \Longrightarrow f(s, \pi(s)) \in f^{\pi}(\omega)$ ". One simple way to incorporate this type of guarantees into our framework is by producing bounds that hold with probability $\alpha^{K}$. If $\alpha$ is close to 1 and $K$ is relatively small, these bounds may still be informative. Also note that we only need to be able to compute $f^{\pi}(\omega)$ for $\omega \in \Omega_{k}$. That is, we do not need to have good transition bounds for parts of the state space that we are certain our policy will not visit. In the future, we plan to work more closely with practitioners in applied fields, and identify what kind of guarantees they are able to provide about the behavior of the systems in which they are interested.

Another interesting area of investigation is analyzing the interplay between the observed transitions and the policy $\pi$ that is evaluated. As we discussed in Section 3.4, the multi-partition bounds become tighter the more transitions there are with starting states close to the regions in $\Omega$, the more the actions for those transitions are similar to those that $\pi$ would take, and also depending on the nature of $\pi$. While these effects are difficult to quantify theoretically, a comprehensive empirical analysis would further the understanding of the multi-partition method's scope of applicability. We also discussed in Section 3.4 how the multi-partition method may suffer from the curse of dimensionality; we would like to further investigate possibilities for addressing this issue.

In this paper we focused solely on producing tight bounds. In the future, we plan to also look at how the bounds produced can be used for selecting what policy to implement. The problem of computing policies that maximize a lower bound on the value function has received a fair amount of attention. Nilim and El Ghaoui (2005) and Delage and Mannor (2009) address this problem in finite state spaces from a game theoretic perspective. In infinite state spaces, Fonteneau et al. (2010) compute lower-boundmaximizing policies by using lower bounds computed using the Lipschitz continuity assumptions (as described in Section 3), while Ermon et al. (2010) address a similar problem in the context of halibut fishery management under convexity assumptions on the reward and the value function. 
In some applications we may be interested in exploratory rather than cautious policies. In such domains, we can use upper bounds computed similarly to the lower bounds to derive exploratory policies that maximize the upper bounds - an idea dating back at least to the work of Kaelbling (1993).

Acknowledgments The authors would like to thank Raphael Fonteneau for providing his implementation of the algorithm described in Fonteneau et al. (2010), and Arthur Guez for helpful discussions regarding this work. Funding was provided by the National Institutes of Health (grant R21 DA019800) and the NSERC Discovery Grant program.

\section{References}

1. Brunskill, E., Leffler, B., Li, L., Littman, M., Roy, N.: CORL: A continuous-state offsetdynamics reinforcement learner. In: Proceedings of the International Conference on Uncertainty in Artificial Intelligence. pp. 53-61 (2008)

2. Delage, E., Mannor, S.: Percentile Optimization for Markov Decision Processes with Parameter Uncertainty. Operations Research 58(1), 203-213 (Aug 2009)

3. Ermon, S., Conrad, J., Gomes, C., Selman, B.: Playing games against nature: optimal policies for renewable resource allocation. In: Proceedings of The 26th Conference on Uncertainty in Artificial Intelligence (2010)

4. Fonteneau, R., Murphy, S., Wehenkel, L., Ernst, D.: Inferring bounds on the performance of a control policy from a sample of trajectories. In: IEEE Symposium on Adaptive Dynamic Programming and Reinforcement Learning (ADPRL). pp. 117-123 (2009)

5. Fonteneau, R., Murphy, S., Wehenkel, L., Ernst, D.: A cautious approach to generalization in reinforcement learning. In: Proceedings of the Second International Conference on Agents and Artificial Intelligence (ICAART 2010), Valencia, Spain (2010)

6. Kaelbling, L.P.: Learning in embedded systems. MIT Press (1993)

7. Kakade, S., Kearns, M., Langford, J.: Exploration in Metric State Spaces. In: International Conference on Machine Learning. vol. 20, p. 306 (2003)

8. Nilim, A., El Ghaoui, L.: Robust Control of Markov Decision Processes with Uncertain Transition Matrices. Operations Research 53(5), 780-798 (Sep 2005) 